ISSN 0103-9954

\title{
INFLUÊNCIA DE ESTIMATIVAS DE PRODUÇÃO DE MADEIRA EM PROCESSOS DE REGULAÇÃO FLORESTAL UTILIZANDO PROGRAMAÇÃO LINEAR
}

\section{INFLUENCE OF WOOD YIELD ESTIMATIONS IN FOREST REGULATION PROCESSES USING LINEAR PROGRAMMING}

\author{
Gilson Fernandes da Silva ${ }^{1}$ Helio Garcia Leite ${ }^{2}$ Carlos Pedro Boechat Soares ${ }^{3}$ Márcio Lopes da Silva ${ }^{4}$
}

\section{RESUMO}

Este trabalho teve como principal objetivo avaliar as conseqüências do uso de estimativas de produção oriundas de diferentes modelos de crescimento e produção em um modelo de Programação Linear aplicado à regulação florestal. Assim, utilizando dados de um inventário florestal contínuo, obtiveram-se diferentes estimativas da produção futura em volume, pelo ajuste de um modelo de produção em função da idade, outro em função da idade e do índice de local e por um terceiro modelo que incluiu, além da idade e do índice de local, a densidade, representada pela área basal por hectare. Testou-se também um modelo baseado nos dados de inventário florestal contínuo que utiliza os dados de volume de um período para fazer projeções lineares da produção para o período seguinte. Em seguida, um problema de regulação florestal simplificado foi idealizado e resolvido pelo modelo I por meio de Programação Linear, utilizando dados oriundos dos quatro modelos de predição do volume. Ao final, concluiu-se: a) que modelos de regulação florestal, alimentados por estimativas provenientes de diferentes modelos de produção, quando resolvidos por Programação Linear, resultam em diferentes maneiras de se conduzir a floresta; b) que a matriz de coeficientes tecnológicos, alimentada por diferentes dados de produção e para uma mesma função objetivo, afeta, de maneira significativa, o processo de tomada de decisão.

Palavras-chave: modelos de crescimento e produção, modelo de gerenciamento, otimização.

\section{ABSTRACT}

The main objective of this work was to evaluate the consequences of the use of yield and growth estimates from different prediction yield and growth models in a Linear Programming model applied to forest regulation. Thus, using data of continuous forest inventory, the yield estimation in future volume was obtained using a yield model based on age, another one based on age and site index, and a third model that used age and site index besides density, which was represented by the basal area per hectare. Also a model based on data of continuous forest inventory which uses data of volume on a period of time to make linear projections of yield for the next period was tested. Next, a simplified forest regulation problem was proposed and solved by the model I by means of Linear Programming, using data from the four volume prediction models. At the end, the conclusion was: a) that models of forest regulation, supplied with estimates from different yield models, when solved by Linear Programming, result in different ways of treating the forests; b) that the matrix of technological coefficients, supplied with different yield data and for a same objective function affects in a significant way the decision making process.

Key words: yield and growth models, management model, optimization.

\section{INTRODUÇÃO}

Modelos de Programação Matemática, mais especificamente modelos de Programação Linear, são alimentados por uma série de dados, dentre os quais possuem especial importância o vetor de custos, o vetor de requisitos e a matriz de coeficientes tecnológicos. De acordo com Bregalda et al. (1988), na formulação

1. Engenheiro Florestal, Dr., Professor do Centro de Ciências Agrárias, Universidade Federal do Espirito Santo, Alto Universário, Caixa Postal 16, CEP 29500-000, Alegre (ES). gfsilva2000@ yahoo.com

2. Engenheiro Florestal, Dr., Professor do Departamento de Engenharia Florestal, Universidade Federal de Viçosa, CEP 36571-000, Viçosa (MG). hgleite@ufv.br

3. Engenheiro Florestal, Dr., Professor do Departamento de Engenharia Florestal, Universidade Federal de Viçosa, CEP 36571-000, Viçosa (MG). csoares@ufv.br

4. Engenheiro Florestal, Dr., Professor do Departamento de Engenharia Florestal, Universidade Federal de Viçosa, CEP 36571-000, Viçosa (MG). marlosil@ufv.br

Recebido para publicação em 7/05/2002 e aceito em 12/12/2002. 
de um modelo de Programação Linear, normalmente os dados disponíveis são quase sempre imprecisos, duvidosos, meras estimativas, estando sujeitos, portanto, a variações. Dessa forma, ao se encontrar a solução de um problema de Programação Linear, esta deverá ser criticada em face das variações que pode sofrer o modelo. A esse estudo, denomina-se estudo de pós-otimização, o qual pode ser desenvolvido por meio da análise de sensibilidade das respostas, com base em variações discretas conhecidas em $A, b$ ou $c$, em que $A$ representa a matriz de coeficientes tecnológicos, $b$ o vetor de requisitos e $c$ o vetor de custos.

Em se tratando de modelos de Programação Matemática aplicados ao planejamento florestal, o mesmo raciocínio pode ser estabelecido, ou seja, respostas encontradas para o planejamento florestal devem ser criticadas, dadas as incertezas que cercam os dados que alimentam os modelos de planejamento florestal. Dentre tais dados, destacam-se grandemente os de volume, associados tanto ao vetor de custos quanto à matriz de coeficientes tecnológicos (Dykstra, 1984; Clutter et al., 1983; Davis e Johnson, 1987). Analisando o impacto da estocasticidade das informações em um modelo de planejamento florestal, Volpi et al. (1999) concluíram que as variáveis com maior impacto nos valores da função objetivo foram primeiramente os preços dos produtos, seguidos pelos coeficientes de produção, pelos custos de manejo e pelas demandas obrigatórias dos produtos.

Embora se saiba da importância da prognose em modelos de planejamento florestal, pouco se tem feito para estudar os seus impactos nas soluções desses modelos. Dessa forma, a predição da produção tem sido feita, muitas vezes, sem qualquer critério, desprezando-se variáveis importantes, como índice de local, densidade inicial, desbastes realizados, entre outras. Por outro lado, Gomes et al. (1998) concluíram que a densidade inicial e o índice de local influenciam de maneira decisiva a rentabilidade econômica máxima da floresta para qualquer idade de corte. Ainda, em muitas situações, não há preocupação dos usuários desses modelos em associar determinado modelo de prognose ao objetivo ao qual se destina, talvez pela falta de acesso a critérios claros e de fácil obtenção para que essa associação possa ser feita.

Tendo em vista o exposto, este trabalho teve como principal objetivo avaliar as conseqüências do uso de modelos de prognose em um modelo de Programação Linear aplicado à regulação florestal.

\section{MATERIAL E MÉTODOS}

Procurando alcançar o objetivo proposto, elaborou-se um problema de regulação florestal simplificado que possui número de variáveis suficientes para ilustrar as situações que se deseja.

\section{Descrição do problema}

Uma empresa florestal deseja elaborar um plano de manejo para uma floresta equiiânea para os próximos oito anos (horizonte de planejamento), com vistas a atender suas necessidades de matéria-prima para produção de celulose, tendo como principal objetivo maximizar o retorno econômico. Na Tabela 1, são apresentadas informações de área, idade atual e classe de produtividade das unidades de manejo consideradas.

TABELA 1: Descrição das unidades de manejo em termos de área, idade atual e classe de produtividade, expressa pelo índice de local (S), em metros.

TABLE 1: Description of the management units in terms of area, current age and productivity class, expressed for the site index (S), in meters.

\begin{tabular}{lcc|c}
\hline Unidade de Manejo & Idade Atual (anos) & Área (ha) & S (m) \\
\hline 1 & 0 & 50,00 & 16 \\
2 & 1 & 85,00 & 16 \\
3 & 2 & 50,00 & 24 \\
4 & 3 & 50,00 & 24 \\
5 & 4 & 90,00 & 24 \\
6 & 5 & 35,00 & 24 \\
7 & 6 & 35,00 & 32 \\
8 & 7 & 60,00 & 32 \\
\hline
\end{tabular}

Propositalmente, para ilustrar o efeito das classes de produtividade no modelo de regulação, foram selecionadas unidades de manejo em plantios do híbrido Eucalyptus grandis x Eucalyptus urophylla em classes de baixa, média e alta produtividade, expressas pelos índices de local (S) 16, 24 e 32 metros, 
respectivamente, para uma idade índice de 60 meses.

Além das informações contidas na Tabela 1, as seguintes informações devem ser consideradas na formulação do problema:

a) Possíveis atividades incluem: corte seguido de imediata regeneração via plantio;

b) Idades mínima e máxima de corte são de 5 e 7 anos, respectivamente;

c) Operações de corte ocorrem uma vez a cada ano, no meio do ano; e

d) A demanda anual do mercado por madeira para celulose é de $15.000,00 \mathrm{~m}^{3} /$ ano.

\section{Estimação da produção para fins de regulação}

A prognose da produção é necessária em modelos de tomada de decisão para regulação da floresta, tanto na maximização da função objetivo como no atendimento de algumas das restrições. Entretanto, diferentes são os modelos de crescimento e produção que poderiam ser utilizados para fornecer estimativas da produção, de modo a alimentar o modelo de regulação. Especificamente neste trabalho, foram considerados os seguintes modelos, os quais são amplamente utilizados em trabalhos científicos, bem como em empresas florestais:

Modelo em função da idade (Função Logística):

$$
V=\frac{\beta_{0}}{1+\beta_{1} \cdot e^{\left(-\beta_{2} \cdot I\right)}} \cdot \varepsilon
$$

Modelo em função da idade e do índice de local:

$$
V=e^{\left(\beta_{0}+\frac{\beta_{1}}{I . S}\right) \varepsilon}
$$

Modelo em função da idade, do índice de local e da área basal (Clutter, 1963):

$\operatorname{Ln}\left(V_{2}\right)=\beta_{0}+\beta_{1}\left(1 / I_{2}\right)+\beta_{2} S+\beta_{3} \operatorname{Ln}\left(G_{2}\right)+\varepsilon$

Sendo $\operatorname{Ln}\left(G_{2}\right)=\operatorname{Ln}\left(G_{1}\right)\left(I_{1} / I_{2}\right)+\alpha_{o}\left(1-I_{1} / I_{2}\right)+\alpha_{1}\left(1-I_{1} / I_{2}\right) S+\varepsilon$

Em que: $V=$ volume, em $\mathrm{m}^{3} ; I=$ idade, em meses; $e=$ exponencial; $S=$ índice de local, em $\mathrm{m} ; \mathrm{Ln}=$ logaritmo neperiano; $V_{2}=$ volume futuro, em m $\mathrm{m}^{3} / \mathrm{ha} ; G_{1}=$ área basal corrente, $\mathrm{em} \mathrm{m} \mathrm{m}^{2} / \mathrm{ha} ; G_{2}=$ área basal futura, $\mathrm{em} \mathrm{m}^{2} / \mathrm{ha} ; I_{l}=$ idade corrente, em meses; $I_{2}=$ idade futura, em meses; $\beta_{i} e \alpha_{j}(\mathrm{i}=0,1,2,3$ e j = 0,1) = parâmetros dos modelos; e $\varepsilon=$ erro aleatório.

O modelo 1, também conhecido como Função Logística, tem como variável dependente apenas a idade e foi ajustado para cada uma das classes de produtividade determinadas. A estimação futura da produção em volume para o modelo 2 foi obtida pela substituição das variáveis idade e índice de local, de acordo com as idades futuras determinadas. Já para o modelo 3, a estimação da produção futura em volume e área basal foi obtida conforme proposto por Clutter (1963) e utilizado por Sulivan e Clutter (1972), Trevisol Júnior (1985) e Campos et al. (1996). O modelo 3 foi ajustado pelo método de mínimos quadrados em dois estágios. Os demais modelos foram ajustados pelo método de mínimos quadrados ordinários.

Além dos modelos 1, 2 e 3, testou-se também um modelo baseado nos dados de inventário florestal contínuo que utiliza os dados de volume de um período para fazer projeções lineares da produção para o período seguinte. Esse método foi empregado por Couto e Bastos (1986) que o denominaram de Equações Lineares Simultâneas. Para maior facilidade na discussão dos resultados, esse modelo será tratado neste trabalho, a partir de então, como modelo de Projeção Linear, o qual pode ser assim representado:

$$
V_{(i+1)}=\beta_{0}+\beta_{1} \cdot V_{i+} \varepsilon
$$

Em que: $V_{i}=$ volume atual medido na idade $\mathrm{i}, \mathrm{em} \mathrm{m}^{3} / \mathrm{ha} ; V_{(i+1)}=$ volume projetado para a idade $(\mathrm{i}+1)$, em $\mathrm{m}^{3} / \mathrm{ha} ; \beta_{i}(\mathrm{i}=$ $0,1)=$ parâmetros do modelo; $\varepsilon=$ erro aleatório.

Os dados utilizados para ajuste dos modelos foram oriundos da empresa COPENER FLORESTAL LTDA, localizada no norte da Bahia. Foram utilizados dados de 177 parcelas permanentes em plantios do híbrido Eucalyptus grandis x Eucalyptus urophylla, com idades entre 28 e 90 meses, sendo as medições e remedições feitas aos 30, 42, 54, 66, 78 e 90 meses. Para o modelo 5, foram ajustadas três equações lineares, sendo utilizados os volumes aos 54, 66 e 78 meses para se projetar os volumes para as idades de 66, 78 e 90 meses, respectivamente.

Ciência Florestal, v. 13, n. 1, 2003 
Para avaliar a precisão dos modelos propostos, obteve-se o coeficiente de determinação $\left(\mathrm{R}^{2}\right)$ e o erro-padrão das estimativas relativo $\left(\mathrm{S}_{\mathrm{y} . \mathrm{x}} \%\right)$ para cada uma das equações ajustadas. Foi feita também uma análise residual para os volumes estimados para as oito unidades de manejo apresentadas na Tabela 1. Esse procedimento foi adotado com base no objetivo principal do trabalho, que é avaliar a influência de estimativas de produção em modelos de Programação Linear para regulação florestal. Como apenas os dados de volume das oito unidades de manejo selecionadas foram utilizados como input no modelo de Programação Linear, procurou-se então avaliar a precisão apenas desses dados por meio de análise de resíduo, em vez de se avaliar a precisão da predição para todos os dados utilizados no ajuste, como usualmente é feito.

\section{Formulação do problema pelo modelo de Programação Linear (PL)}

O problema proposto foi formulado como um problema de Programação Linear clássico. Foi empregado o modelo I, proposto originalmente por Kidd et al. (1966), citados por Dykstra (1984), recebendo essa denominação por Johnson e Scheurman (1977). Nessa formulação, a variável de decisão $X_{i j}$ representa a fração do povoamento $i$, que deverá ser assinalada na prescrição $j$, conforme descrito por Ribeiro (1996).

Com base no exposto, prescrições de manejo foram sugeridas para o problema em questão, conforme apresentado na Tabela 2. Desse modo, a variável de decisão $X_{11}$ representa a área em hectares a TABELA 2: Prescrições de manejo viáveis para um horizonte de planejamento de oito anos.

TABLE 2: Viable management prescriptions for a planning horizon of eight years.

\begin{tabular}{|c|c|c|c|c|c|c|c|c|c|c|c|}
\hline \multirow{2}{*}{$\begin{array}{l}\text { Prescrições } \\
\text { de Manejo }\end{array}$} & \multirow{2}{*}{$\begin{array}{l}\text { Idade } \\
\text { Atual }\end{array}$} & \multirow{2}{*}{$\begin{array}{c}\text { Rotações } \\
\text { em anos }\end{array}$} & \multicolumn{8}{|c|}{ Horizonte de Planejamento } & \multirow{2}{*}{$\begin{array}{l}\text { Idade } \\
\text { Final }\end{array}$} \\
\hline & & & 0 & 1 & 2 & 3 & 4 & 5 & 6 & 7 & \\
\hline$\overline{\mathrm{X}_{11}}$ & 0 & 5 & & & & & & & & & 3 \\
\hline $\mathrm{X}_{12}$ & 0 & 6 & & & & & & & & & 2 \\
\hline $\mathrm{X}_{13}$ & 0 & 7 & & & & & & & & & 1 \\
\hline $\mathrm{X}_{21}$ & 1 & 5 & & & & & & & & & 4 \\
\hline$X_{22}$ & 1 & 6 & & & & & & & & & 3 \\
\hline$X_{23}$ & 1 & 7 & & & & & & & & & 2 \\
\hline $\mathrm{X}_{31}$ & 2 & 5 & & & & & & & & & 5 \\
\hline$X_{32}$ & 2 & 6 & & & & & & & & & 4 \\
\hline$X_{33}$ & 2 & 7 & & & & & & & & & 3 \\
\hline$X_{41}$ & 3 & $5-5$ & & & & & & & & & 1 \\
\hline$X_{42}$ & 3 & 6 & & & & & & & & & 5 \\
\hline $\mathrm{X}_{43}$ & 3 & 7 & & & & & & & & & 4 \\
\hline $\mathrm{X}_{51}$ & 4 & $5-5$ & & & & & & & & & 2 \\
\hline $\mathrm{X}_{52}$ & 4 & $5-6$ & & & & & & & & & 1 \\
\hline $\mathrm{X}_{53}$ & 4 & $6-5$ & & & & & & & & & 1 \\
\hline $\mathrm{X}_{54}$ & 4 & 7 & & & & & & & & & 5 \\
\hline$X_{61}$ & 5 & $5-5$ & & & & & & & & & 3 \\
\hline$X_{62}$ & 5 & $5-6$ & & & & & & & & & 2 \\
\hline $\mathrm{X}_{63}$ & 5 & $5-7$ & & & & & & & & & 1 \\
\hline $\mathrm{X}_{64}$ & 5 & $6-5$ & & & & & & & & & 2 \\
\hline $\mathrm{X}_{65}$ & 5 & $6-6$ & & & & & & & & & 1 \\
\hline $\mathrm{X}_{66}$ & 5 & $7-5$ & & & & & & & & & 1 \\
\hline $\mathrm{X}_{71}$ & 6 & $6-5$ & & & & & & & & & 3 \\
\hline$X_{72}$ & 6 & $6-6$ & & & & & & & & & 2 \\
\hline $\mathrm{X}_{73}$ & 6 & $6-7$ & & & & & & & & & 1 \\
\hline$X_{74}$ & 6 & $7-5$ & & & & & & & & & 2 \\
\hline$X_{75}$ & 6 & $7-6$ & & & & & & & & & 1 \\
\hline$X_{81}$ & 7 & $7-5$ & & & & & & & & & 3 \\
\hline$X_{82}$ & 7 & $7-6$ & & & & & & & & & 2 \\
\hline$X_{83}$ & 7 & $7-7$ & & & & & & & & & 1 \\
\hline
\end{tabular}

Em que: = Estoque de colheita;

Estoque de crescimento. 
ser cortada no povoamento 1 aos cinco anos de idade, a variável de decisão $X_{12}$, a área a ser cortada no povoamento 1 aos seis anos de idade, e assim sucessivamente. Para determinação das prescrições, foram consideradas como idades de corte, mínima e máxima, cinco e sete anos respectivamente.

Levando-se em conta que foram propostos quatro maneiras diferentes de obter a produção, e sendo a produção um componente importante do modelo de otimização, foram elaborados quatro diferentes modelos de otimização, cada um associado a um modelo de prognose, conforme apresentado no item anterior.

\section{Função objetivo}

Considerou-se como objetivo do empreendedor a maximização do Benefício Periódico Equivalente (BPE) global da floresta, definindo-se a seguinte função objetivo:

$$
\operatorname{Max} B P E_{G}=\sum_{i=1}^{M} \sum_{j=1}^{N} C_{i j} X_{i j}
$$

Em que: $B P E_{G}=$ benefício periódico equivalente (BPE) global; $C_{i j}=$ benefício periódico equivalente de cada hectare do povoamento $i$, manejado sob a alternativa de manejo $j ; X_{i j}=$ fração de área (ha) do povoamento $i$ assinalada na alternativa de manejo $j ; M=$ número total de unidades de manejo; $N=$ número total de alternativas de manejo do i-ésimo povoamento.

Para obtenção do Benefício Periódico Equivalente (BPE), empregou-se a metodologia apresentada em Rezende e Oliveira (1993) e Gomes et al. (1998). Desse modo, o BPE foi obtido pela seguinte expressão:

$$
B P E=\frac{V P L . i}{\left[1-(1+i)^{-n}\right]}
$$

Em que: $B P E=$ benefício periódico equivalente, em $\mathrm{R} \$ / \mathrm{ha} / \mathrm{ano} ; V P L=$ valor presente líquido, em $\mathrm{R} \$ / \mathrm{ha} ; i=$ taxa de desconto; $n=$ duração do projeto, em anos.

Consideraram-se ainda a taxa de desconto (i) de $7 \%$ a.a., o preço da madeira posto na fábrica igual a $\mathrm{R} \$ 42,00 / \mathrm{m}^{3}$ e os valores de custos médios apresentados na Tabela 3 para o cálculo do BPE.

Conforme ressaltado anteriormente, foram propostos quatro modelos de otimização. Para cada um deles, $C_{i j}$ variou de acordo com as prognoses feitas pelos modelos propostos.

TABELA 3: Custos médios das principais atividades e o ano de ocorrência.

\begin{tabular}{|c|c|c|}
\hline Atividade & Custo $(\mathrm{R} \$ / \mathrm{ha})$ & Ano de Ocorrência \\
\hline Implantação & 1040,12 & 0 \\
\hline \multirow[t]{7}{*}{ Manutenção } & 97,12 & 0 \\
\hline & 168,85 & No ano 1 e no $1^{\circ}$ ano após reforma \\
\hline & 79,65 & No ano 2 e no $2^{\underline{0}}$ ano após reforma \\
\hline & 79,65 & No ano 3 e no $3^{\circ}$ ano após reforma \\
\hline & 40,25 & Do $4^{\circ}$ até o ano do $1^{\circ}$ corte, o mesmo após reforma \\
\hline & 140,13 & No ano do $1^{\circ}$ corte \\
\hline & 140,13 & No ano do $2^{\underline{0}}$ corte \\
\hline Reforma & 871,27 & Ano zero do $2^{\underline{o}}$ ciclo \\
\hline Exploração $\left(\mathrm{R} \$ / \mathrm{m}^{3}\right)$ & 10,91 & Nos anos do $1^{\underline{o}}$ e $2^{\underline{o}}$ corte \\
\hline Custo anual da terra & $1200 \times \mathrm{i}$ & Anualmente \\
\hline
\end{tabular}

TABLE 3: Average costs of the main activities and the year of occurrence.

\section{Restrição de área disponível para colheita}

Essa é a restrição que determina a disponibilidade total de área de cada unidade de manejo florestal e é imprescindível, uma vez que impõe que a soma das áreas de uma mesma unidade de manejo submetida a diferentes alternativas de corte seja igual à área total de cada unidade de manejo, sendo:

$$
\sum_{i=1}^{M} \sum_{j=1}^{N} X_{i j} \leq A_{i}(i=1, \ldots, M e j=1, \ldots, N)
$$

Em que: $A_{i}=$ área da i-ésima unidade de manejo no início do horizonte de planejamento. 
Assim, a restrição pode ser escrita da seguinte forma:

$$
\begin{aligned}
& X_{11}+X_{12}+X_{13} \leq 50 \\
& X_{21}+X_{22}+X_{23} \leq 85 \\
& X_{31}+X_{32}+X_{33} \leq 50 \\
& X_{41}+X_{42}+X_{43} \leq 50 \\
& X_{51}+X_{52}+X_{53}+X_{54} \leq 90 \\
& X_{61}+X_{62}+X_{63}+X_{64}+X_{65}+X_{66} \leq 35 \\
& X_{71}+X_{72}+X_{73}+X_{74}+X_{75} \leq 35 \\
& X_{81}+X_{82}+X_{83} \leq 60
\end{aligned}
$$

\section{Imposição de cotas ou produções anuais}

Essa restrição visa a estabelecer uma produção periódica de volume de madeira de modo a atender a uma demanda pré-estabelecida.

Considerando-se $V_{k}$ o volume total removido no período $k$, então:

$$
V_{k}=\sum_{i=1}^{M} \sum_{j=1}^{N} V_{i j k} X_{i j}\{k=0,1, \ldots, H-2\}
$$

Em que: $V_{i j k}=$ volume $\left(\mathrm{m}^{3} /\right.$ há$)$ produzido pelo i-ésimo povoamento assinalado na j-ésima alternativa de manejo, no início do período $k ; V_{k}=$ volume total $\left(\mathrm{m}^{3}\right)$ removido no período $k$, representando a demanda a ser atendida no período $\mathrm{k} ; H=$ horizonte de planejamento.

Para o problema proposto, a demanda mínima do mercado por madeira é de $15.000,00 \mathrm{~m}^{3} / \mathrm{ano}$. Contudo, deseja-se também que a produção de madeira não exceda $20.000,00 \mathrm{~m}^{3} /$ ano, prevendo-se um excesso de produção que poderia acarretar dificuldades na sua estocagem e venda. Desse modo, essas restrições foram assim representadas:

$$
15.000 \leq \sum_{i=1}^{M} \sum_{j=1}^{N} V_{i j k} X_{i j} \leq 20.000 \quad\{k=0,1, \ldots, H-2\}
$$

Em que: $V_{i j k}$ variou de acordo com os modelos de prognose propostos.

Cabe citar finalmente que os modelos de PL foram resolvidos empregando-se o algoritmo Simplex, implementado no software LINDO/PC, versão 6.01 (1997).

\section{RESULTADOS E DISCUSSÃO}

\section{Ajuste dos modelos de produção}

Inicialmente, ajustaram-se os modelos de produção propostos, de modo a gerar as tabelas de produção com o propósito de fornecer dados para o modelo de otimização. As equações ajustadas bem como as suas respectivas medidas de precisão foram:

Modelo 1 (Função Logística):

$$
\begin{aligned}
& \hat{V}=\frac{133,27440}{1+17,01338 \cdot e^{(-0,05930 . I)}}, \mathrm{S}=16 \mathrm{~m} ; \mathrm{R}^{2}=69,79 ; \mathrm{s}_{\mathrm{y} . \mathrm{x}}(\%)=23,00 ; \\
& \hat{V}=\frac{13,49693}{1-0,82100 \cdot e^{(0,00160 . I)}}, \quad \mathrm{S}=24 \mathrm{~m} ; \mathrm{R}^{2}=52,28 ; \mathrm{s}_{\mathrm{y} . \mathrm{x}}(\%)=33,43 ; \\
& \hat{V}=\frac{304,38860}{1+22,77638 . e^{(-0,07707 . I)}}, \mathrm{S}=32 \mathrm{~m} ; \mathrm{R}^{2}=80,15 ; \mathrm{s}_{\mathrm{y} \cdot \mathrm{x}}(\%)=16,38 .
\end{aligned}
$$

Modelo 2:

$$
\hat{V}=e^{\left(6,3225-\frac{1834,6900}{I . S}\right)}, \quad \mathrm{R}^{2}=77,68 ; \quad \mathrm{s}_{\mathrm{y} . \mathrm{x}}(\%)=26,00 .
$$


Modelo 3 (Modelo de Clutter):

$$
\begin{aligned}
& \operatorname{Ln}\left(G_{2}\right)=\operatorname{Ln}\left(G_{1}\right) \cdot\left(I_{1} / I_{2}\right)+2,28572 .\left(1-I_{1} / I_{2}\right)+0,04277 \cdot\left(1-I_{1} / I_{2}\right) \cdot S ; \mathrm{R}^{2}=92,47 ; \quad \mathrm{s}_{\mathrm{y} . \mathrm{x}}(\%)=15,28 ; \\
& \operatorname{Ln}\left(V_{2}\right)=1,6574-21,7339.1 / I_{2}+0,0296 . S+1,0930 . \operatorname{Ln}\left(G_{2}\right) ; \quad \mathrm{R}^{2}=93,26 ; \quad \mathrm{s}_{\mathrm{y} . \mathrm{x}}(\%)=14,32 .
\end{aligned}
$$

Projeção Linear:

$$
\begin{array}{lll}
\hat{V}_{66}=-11,94900+1,29765 . V_{54} ; & \mathrm{R}^{2}=94,55 ; & \mathrm{s}_{\mathrm{y} \cdot \mathrm{x}}(\%)=9,38 ; \\
\hat{V}_{78}=8,275799+1,142961 . V_{66} ; & \mathrm{R}^{2}=96,52 ; & \mathrm{s}_{\mathrm{y} \cdot \mathrm{x}}(\%)=7,27 ; \\
\hat{V}_{90}=15,18247+1,00176 . V_{78} ; & \mathrm{R}^{2}=87,49 ; & \mathrm{s}_{\mathrm{y} \cdot \mathrm{x}}(\%)=14,17 .
\end{array}
$$

De acordo com as estatísticas apresentadas, pode-se observar que as equações ajustadas para o modelo de Projeção Linear mostraram ser, de maneira geral, as mais precisas, seguidas pelas equações ajustadas para o modelo 3 e pela equação ajustada para o modelo 2, enquanto as equações ajustadas para o modelo 1 mostraram ser, de modo geral, menos precisas, fato já esperado, dado que esse modelo tem como variável independente apenas a idade, o que não é, na maioria das vezes, suficiente para explicar satisfatoriamente as variações na produção. Reforçando essa idéia, o modelo 3 que além da idade considera a área basal inicial e o índice de local, proporcionou melhores resultados, ou seja, considerou variáveis importantes além da idade, como a área basal inicial e o índice de local, para explicar as variações ocorridas na produção. Por outro lado, poder-se questionar o fato do modelo de projeção linear apresentar apenas uma variável independente e mesmo assim ter sido o mais preciso. Para se compreender este resultado, basta observar que esse modelo tem como variável independente o volume na idade imediatamente anterior que tem alta correlação com o volume atual. Entretanto, embora isso ocorra, a obtenção dessa variável independente é algo difícil, pois implicaria em inventários constantes em todas as unidades de manejo, o que, na prática, seria inviável.

Na Figura 1, é apresentada uma análise gráfica dos resíduos das predições de volume para os modelos propostos. Essa análise incluiu apenas as predições de volume das unidades de manejo consideradas, e não de todos os dados utilizados no ajuste das equações, conforme justificado no item Material e Métodos. Essa Figura confirma as estatísticas apresentadas para as equações dos modelos apresentados, ou seja, as equações ajustadas para o modelo de Projeção Linear mostraram ser, de maneira geral, as mais precisas, seguidas pelas equações ajustadas para o modelo 3 e pela equação ajustada para o modelo 2, enquanto as equações ajustadas para o modelo 1 mostraram ser, de modo geral, menos precisas.

Embora o modelo de Projeção Linear se tenha apresentado ligeiramente mais preciso neste estudo que o modelo 3, conforme já discutido, quando se pensa em questões de manejo, como o processo de regulação florestal apresentado neste trabalho, o modelo de Projeção Linear apresenta sérias restrições. Uma delas é o fato de que esse modelo se baseia numa lógica muito simplista da realidade florestal ao supor que a taxa de crescimento de uma floresta eqüiânea seja função apenas do volume no ano anterior e linear ao longo dos anos, desprezando variáveis importantes no manejo florestal, como o índice de local e a densidade, aumentando assim o seu grau de empirismo. Outra séria restrição do modelo de Projeção Linear está no fato de que é necessário que se saiba a produção no ano anterior para se predizer a produção na idade desejada, obrigando a existência de parcelas do inventário florestal contínuo em todas as unidades de manejo em todas as idades, o que na prática seria inviável.

Uma vez ajustados os modelos propostos, foi gerada uma tabela de produção, conforme apresentado na Tabela 4. Essa tabela mostra as produções médias esperadas, de acordo com cada modelo proposto, para as idades de 66, 78 e 90 meses, as quais foram utilizadas no modelo de regulação elaborado. Cabe ressaltar que, para obter as produções pelo modelo 3, utilizou-se, para todas as unidades de manejo, a área basal inicial $\left(\mathrm{G}_{1}\right)$ aos 54 meses, e para os modelos de Projeção Linear, as produções observadas no período anterior para obter as produções no período desejado, conforme mostra a Tabela 4. 
Modelo 1

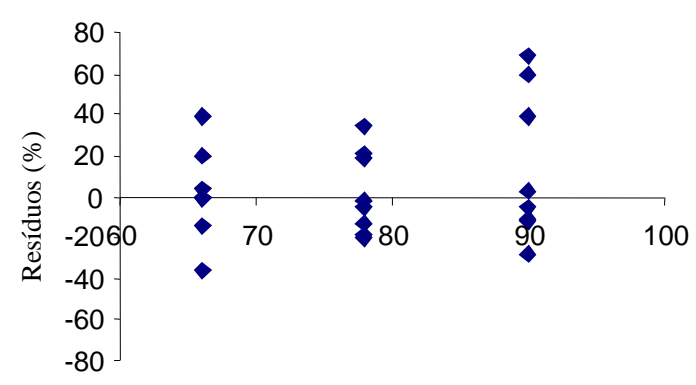

Idade (meses)

Modelo 2

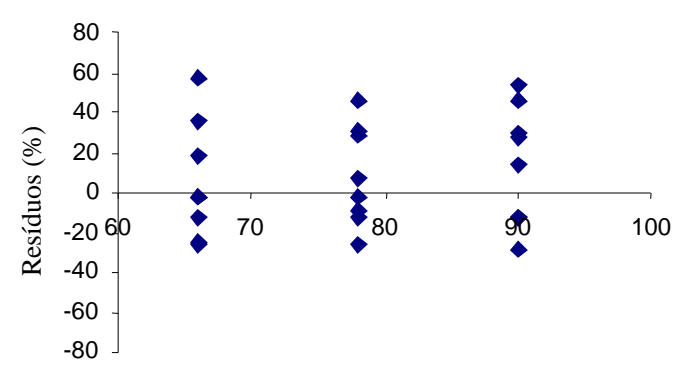

Idade (meses)

Modelo 3

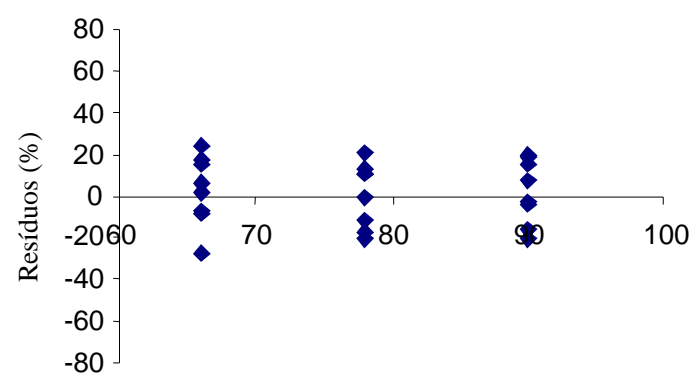

Idade (meses)

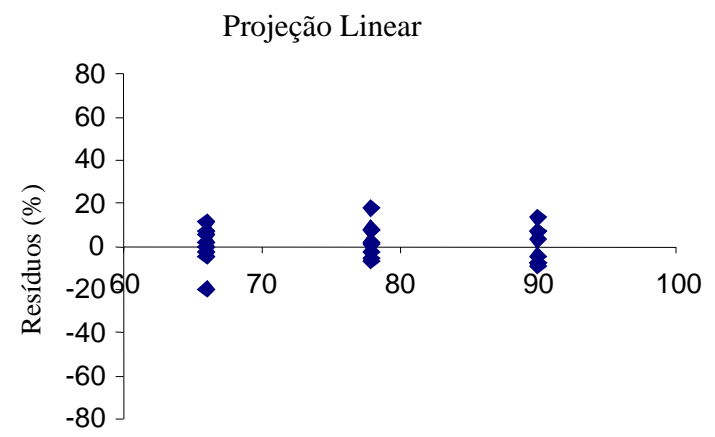

Idade (meses)

FIGURA 1: Resíduo percentual na predição do volume em função da idade para os quatro modelos de predição de volume propostos.

FIGURE 1: Percentile residue in the prediction of the volume as a function of the age for the four models of volume prediction proposed. 
TABELA 4: Tabela de produção, mostrando os volumes por hectare $\left(\mathrm{m}^{3} / \mathrm{ha}\right)$ para os modelos propostos, considerando a área basal inicial $\mathrm{G}_{1}\left(\mathrm{~m}^{2} / \mathrm{ha}\right)$ aos 54 meses, com idades variando de 66 até 90 meses, sendo $\mathrm{S}$ o índice de local em metros.

TABLE 4: Yield volume table, showing the volumes for hectare $\left(\mathrm{m}^{3} / \mathrm{ha}\right)$ for the proposed models, considering the initial basal area $\mathrm{G}_{1}\left(\mathrm{~m}^{2} / \mathrm{ha}\right)$ at the 54 months, with ages varying from 66 to 90 months, being $\mathrm{S}$ the site index in meters.

\begin{tabular}{|c|c|c|c|c|c|c|c|c|}
\hline \multirow{2}{*}{$\begin{array}{c}\text { Unidade } \\
\text { de } \\
\text { Manejo }\end{array}$} & \multirow{2}{*}{$\begin{array}{c}\text { Idade } \\
\text { (meses) }\end{array}$} & \multirow[b]{2}{*}{$\begin{array}{c}\mathrm{S} \\
(\mathrm{m})\end{array}$} & \multirow{2}{*}{$\begin{array}{c}\mathrm{G}_{1} \\
\left(\mathrm{~m}^{2} / \mathrm{ha}\right)\end{array}$} & \multirow[b]{2}{*}{$\begin{array}{l}\text { Vol. Obs. } \\
\left(\mathrm{m}^{3} / \mathrm{ha}\right)\end{array}$} & \multicolumn{4}{|c|}{ Volume $\left(\mathrm{m}^{3} / \mathrm{ha}\right)$} \\
\hline & & & & & $\begin{array}{c}\text { Projeção } \\
\text { Linear }\end{array}$ & $\begin{array}{c}\text { Modelo } \\
1\end{array}$ & $\begin{array}{c}\text { Modelo } \\
2\end{array}$ & $\begin{array}{c}\text { Modelo } \\
3\end{array}$ \\
\hline 1 & 54 & 16 & 10,74 & 84,59 & - & - & - & - \\
\hline 1 & 66 & 16 & - & 100,15 & 97,82 & 99,49 & 98,01 & 91,43 \\
\hline 1 & 78 & 16 & - & 131,11 & 122,75 & 114,23 & 128,05 & 104,39 \\
\hline 1 & 90 & 16 & - & 137,25 & 146,52 & 123,19 & 155,77 & 115,05 \\
\hline 2 & 54 & 16 & 10,93 & 86,63 & - & - & - & - \\
\hline 2 & 66 & 16 & - & 100,14 & 100,47 & 99,49 & 98,01 & 92,89 \\
\hline 2 & 78 & 16 & - & 119,89 & 122,73 & 114,23 & 128,05 & 105,80 \\
\hline 2 & 90 & 16 & - & 119,81 & 135,28 & 123,19 & 155,77 & 116,39 \\
\hline 3 & 54 & 24 & 12,10 & 99,70 & - & - & - & - \\
\hline 3 & 66 & 24 & - & 111,04 & 117,42 & 154,15 & 174,90 & 137,93 \\
\hline 3 & 78 & 24 & - & 143,83 & 122,23 & 193,17 & 209,02 & 162,39 \\
\hline 3 & 90 & 24 & - & 154,59 & 159,26 & 260,36 & 238,20 & 183,04 \\
\hline 4 & 54 & 24 & 13,96 & 130,52 & - & - & - & - \\
\hline 4 & 66 & 24 & - & 147,53 & 157,42 & 154,15 & 174,90 & 156,70 \\
\hline 4 & 78 & 24 & - & 163,29 & 176,89 & 193,17 & 209,02 & 180,90 \\
\hline 4 & 90 & 24 & - & 187,10 & 178,76 & 260,36 & 238,20 & 201,00 \\
\hline 5 & 54 & 24 & 13,45 & 111,17 & - & - & - & - \\
\hline 5 & 66 & 24 & - & 129,12 & 132,32 & 154,15 & 174,90 & 151,62 \\
\hline 5 & 78 & 24 & - & 159,17 & 155,85 & 193,17 & 209,02 & 175,93 \\
\hline 5 & 90 & 24 & - & 163,55 & 174,64 & 260,36 & 238,20 & 196,20 \\
\hline 6 & 54 & 24 & 15,57 & 157,03 & - & - & - & - \\
\hline 6 & 66 & 24 & - & 238,85 & 191,82 & 154,15 & 174,90 & 172,75 \\
\hline 6 & 78 & 24 & - & 238,00 & 281,27 & 193,17 & 209,02 & 196,47 \\
\hline 6 & 90 & 24 & - & 274,12 & 253,60 & 260,36 & 238,20 & 215,91 \\
\hline 7 & 54 & 32 & 21,65 & 237,91 & - & - & - & - \\
\hline 7 & 66 & 32 & - & 310,39 & 296,77 & 266,84 & 233,64 & 314,72 \\
\hline 7 & 78 & 32 & - & 360,70 & 363,04 & 288,30 & 267,05 & 358,53 \\
\hline 7 & 90 & 32 & - & 410,37 & 376,51 & 297,80 & 294,55 & 394,49 \\
\hline 8 & 54 & 32 & 21,21 & 237,67 & - & - & - & - \\
\hline 8 & 66 & 32 & - & 267,79 & 296,46 & 266,84 & 233,64 & 308,91 \\
\hline 8 & 78 & 32 & - & 292,87 & 314,35 & 288,30 & 267,05 & 352,92 \\
\hline 8 & 90 & 32 & - & 338,79 & 308,57 & 297,80 & 294,55 & 389,13 \\
\hline
\end{tabular}

\section{Solução do modelo de programação linear}

O modelo matemático de PL pode ser descrito genericamente como:

Objetivo: Max. ou Min. $Z=C_{11} X_{11}+C_{12} X_{12}+\ldots \ldots+C_{i j} X_{i j}$

$$
\text { Sujeito a: } \begin{gathered}
a_{11} X_{11}+a_{12} X_{12}+\ldots \ldots+a_{1 j} X_{1 j} \leq b_{1} \\
a_{21} X_{21}+a_{22} X_{22}+\ldots \ldots+a_{2 j} X_{2 j}=b_{2} \\
\ldots \ldots \ldots \ldots \ldots \ldots \ldots \ldots \ldots \ldots \ldots \ldots \ldots \ldots \\
a_{i 1} X_{i 1}+a_{i 2} X_{i 2}+\ldots \ldots+a_{i j} X_{i j} \geq b_{n}
\end{gathered}
$$

De acordo com a expressão anterior, é fácil notar que os dados de volume oriundos dos quatro modelos de predição podem gerar incertezas tanto no vetor de custos $c$ quanto na matriz de coeficientes tecnológicos $A$. Mesmo o vetor de requisitos $b$ é passível de incertezas. A questão situa-se no fato de que a literatura e mesmo a prática florestal oferecem diversas opções de modelos de produção e nem sempre há o 
cuidado de se avaliar o impacto que a escolha de um ou de outro modelo possa causar na decisão final a ser tomada.

Gomes et al. (1998) destacam, em seu trabalho, a importância em considerar as variáveis índice de local e densidade em modelos de produção e seu impacto direto nos resultados econômicos obtidos pela floresta. Considerando que tanto os resultados econômicos, representados pelo vetor de custos, como as restrições a serem atendidas, representadas pela matriz dos coeficientes tecnológicos e pelo vetor de requisitos, são muito dependentes de dados de volume, há que se ter cuidado especial na escolha de modelos de produção, de modo que a decisão final a ser tomada se aproxime o máximo possível da real solução ótima.

$\mathrm{Na}$ Tabela 5, podem-se observar as soluções para o problema de PL alimentado por diferentes estimativas de produção, segundo os modelos propostos bem como o Custo Reduzido (CR) para as variáveis do problema, o qual informa a redução no valor da função objetivo (FO) para cada unidade de uma variável não-básica que passe a fazer parte da base. Verifica-se, na Tabela 5, que para os

TABELA 5: Respostas obtidas para o modelo de Programação Linear utilizando estimativas de produção oriundas dos diferentes modelos de prognose.

TABLE 5: Answers obtained for the Linear Programming model using yield estimates coming from the different prognosis models.

\begin{tabular}{|c|c|c|c|c|c|c|c|c|}
\hline \multirow{3}{*}{ Variável } & \multicolumn{2}{|c|}{ Projeção Linear } & \multicolumn{2}{|c|}{ Modelo 1} & \multicolumn{2}{|c|}{ Modelo 2} & \multicolumn{2}{|c|}{ Modelo 3} \\
\hline & $\mathrm{FO}=\mathrm{R} \$$ & $2.235,90$ & $\mathrm{FO}=\mathrm{R} \$$ & $57.634,30$ & $\mathrm{FO}=\mathrm{R} \$$ & $8.874,70$ & $\mathrm{FO}=\mathrm{R} \$$ & $50.207,50$ \\
\hline & Área (ha) & $\mathrm{CR}$ & Área (ha) & CR & Área (ha) & CR & Área (ha) & CR \\
\hline $\mathrm{X}_{11}$ & 0,00 & 82,55 & 0,00 & 89,75 & 0,00 & 85,59 & 0,00 & 43,44 \\
\hline$X_{12}$ & 11,95 & 0,00 & 0,00 & 53,93 & 0,00 & 48,19 & 0,00 & 45,63 \\
\hline $\mathrm{X}_{13}$ & 25,60 & 0,00 & 0,00 & 54,11 & 27,14 & 0,00 & 0,00 & 42,96 \\
\hline $\mathrm{X}_{21}$ & 85,00 & 0,00 & 45,48 & 0,00 & 13,26 & 0,00 & 53,85 & 0,00 \\
\hline$X_{22}$ & 0,00 & 100,70 & 0,00 & 59,78 & 7,67 & 0,00 & 27,84 & 0,00 \\
\hline$X_{23}$ & 0,00 & 75,04 & 0,00 & 51,24 & 64,07 & 0,00 & 0,00 & 24,68 \\
\hline $\mathrm{X}_{31}$ & 2,22 & 0,00 & 0,00 & 58,59 & 0,00 & 62,04 & 0,00 & 75,98 \\
\hline $\mathrm{X}_{32}$ & 47,78 & 0,00 & 33,76 & 0,00 & 50,00 & 0,00 & 50,00 & 0,00 \\
\hline$X_{33}$ & 0,00 & 166,94 & 16,24 & 0,00 & 0,00 & 151,04 & 0,00 & 21,42 \\
\hline$X_{41}$ & 50,00 & 0,00 & 34,81 & 0,00 & 36,36 & 0,00 & 40,65 & 0,00 \\
\hline$X_{42}$ & 0,00 & 68,27 & 0,00 & 144,98 & 0,00 & 12,84 & 0,00 & 34,17 \\
\hline $\mathrm{X}_{43}$ & 0,00 & 209,20 & 15,18 & 0,00 & 13,64 & 0,00 & 9,35 & 0,00 \\
\hline $\mathrm{X}_{51}$ & 0,00 & 210,83 & 0,00 & 51,92 & 0,00 & 56,74 & 0,00 & 32,23 \\
\hline$X_{52}$ & 0,00 & 235,57 & 29,69 & 0,00 & 0,00 & 32,18 & 0,00 & 22,43 \\
\hline$X_{53}$ & 5,60 & 0,00 & 2,70 & 0,00 & 27,03 & 0,00 & 13,55 & 0,00 \\
\hline $\mathrm{X}_{54}$ & 84,40 & 0,00 & 57,61 & 0,00 & 62,97 & 0,00 & 76,45 & 0,00 \\
\hline$X_{61}$ & 0,00 & 477,46 & 0,00 & 747,27 & 0,00 & 160,21 & 0,00 & 727,02 \\
\hline$X_{62}$ & 0,00 & 250,31 & 0,00 & 670,98 & 0,00 & 193,86 & 0,00 & 761,05 \\
\hline$X_{63}$ & 0,00 & 493,48 & 0,00 & 572,55 & 0,00 & 194,53 & 0,00 & 787,77 \\
\hline$X_{64}$ & 0,00 & 73,89 & 0,00 & 164,28 & 10,53 & 0,00 & 6,07 & 0,00 \\
\hline$X_{65}$ & 10,33 & 0,00 & 0,00 & 116,53 & 11,91 & 0,00 & 0,00 & 4,18 \\
\hline$X_{66}$ & 24,67 & 0,00 & 35,00 & 0,00 & 12,56 & 0,00 & 28,93 & 0,00 \\
\hline$X_{71}$ & 0,00 & 133,73 & 0,00 & 557,00 & 0,00 & 194,23 & 0,00 & 1470,35 \\
\hline$X_{72}$ & 2,88 & 0,00 & 0,00 & 1556,38 & 0,00 & 278,76 & 0,00 & 1540,90 \\
\hline$X_{73}$ & 0,00 & 210,92 & 0,00 & 719,24 & 0,00 & 309,25 & 0,00 & 1602,42 \\
\hline $\mathrm{X}_{74}$ & 32,12 & 0,00 & 35,00 & 0,00 & 35,00 & 0,00 & 35,00 & 0,00 \\
\hline$X_{75}$ & 0,00 & 55,61 & 0,00 & 35,94 & 0,00 & 2,33 & 0,00 & 21,16 \\
\hline $\mathrm{X}_{81}$ & 50,60 & 0,00 & 40,37 & 0,00 & 60,00 & 0,00 & 39,02 & 0,00 \\
\hline$X_{82}$ & 9,40 & 0,00 & 19,63 & 0,00 & 0,00 & 86,08 & 8,32 & 0,00 \\
\hline $\mathrm{X}_{83}$ & 0,00 & 214,88 & 0,00 & 158,8 & 0,00 & 117,92 & 4,05 & 0,00 \\
\hline
\end{tabular}

Em que: FO = valor obtido para a função objetivo para cada modelo; Área (ha) = área a ser cortada na unidade $i$, segundo a alternativa $j ; \mathrm{CR}=$ custo reduzido $(\mathrm{R} \$)$. 
diferentes modelos de predição do volume, diferentes respostas foram obtidas, com diferentes valores de CR. Considerando que o CR de certa forma atribui um peso à alternativa de manejo considerada, pode-se dizer que, para diferentes modelos de produção, diferentes formas de regular a floresta foram obtidas, tendo as alternativas de manejo recebido diferentes pesos em cada forma de se regular a floresta.

As estimativas de volume, que originaram o maior valor para a função objetivo, foram aquelas oriundas do modelo 2, e o menor valor para a função objetivo está associado ao modelo de Projeção Linear. Esse resultado está de acordo com o esperado, pois, de modo geral, o modelo 2 produziu as expectativas mais otimistas de produção (Tabela 4), o que resultou em um maior valor para a função objetivo associada a esse modelo. O contrário ocorreu com o modelo de Projeção Linear.

As Figuras 2 e 3 permitem comparar de maneira mais ilustrativa as respostas mostradas na Tabela 5. Nessas figuras, verifica-se que cada modelo de predição leva a uma estratégia diferente de corte, especialmente em termos de área a ser cortada.

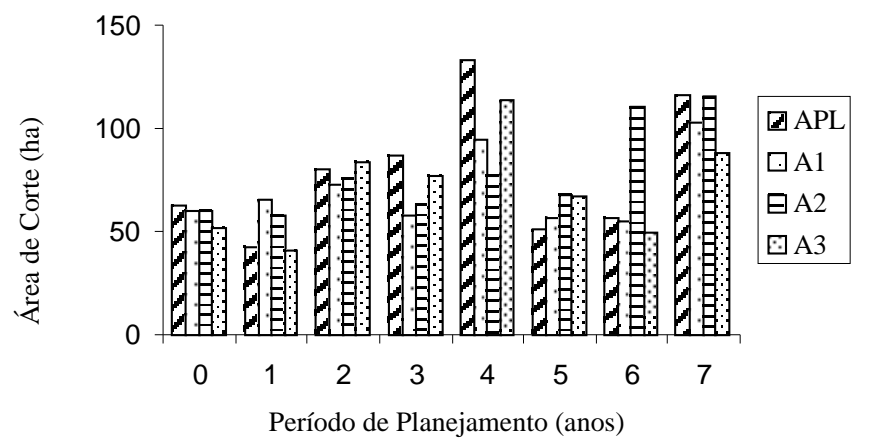

FIGURA 2: Comparação entre os modelos de predição do volume utilizados para regular a floresta, em que APL, A1, A2 e A3 correspondem às áreas anuais cortadas (ha), segundo as soluções obtidas por Programação Linear empregando estimativas de volume dos modelos de Projeção Linear, 1,2 e 3 , respectivamente.

FIGURE 2: Comparison among the models of prediction of the volume used to regulate the forest, in that APL, A1, A2 and A3 correspond to the annual area cut (ha), according to the solutions obtained by Linear Programming using estimates of volume of the models of Linear Projection, 1, 2 and 3, respectively.

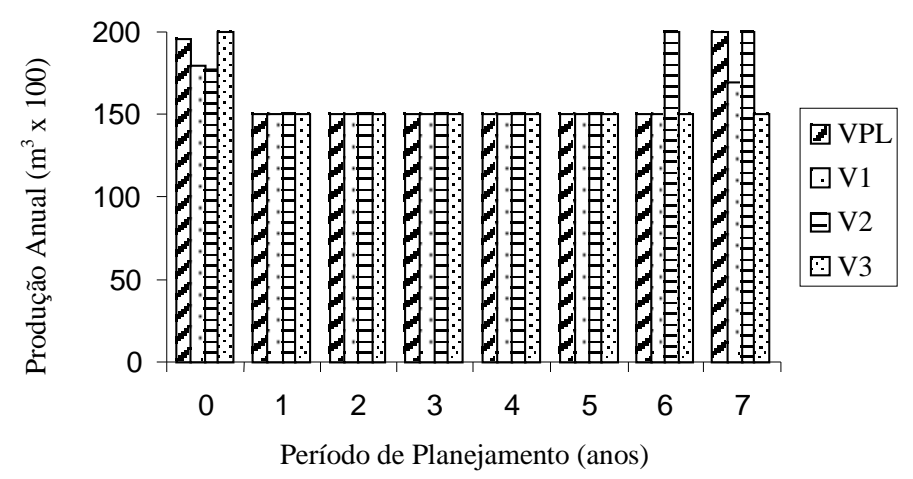

FIGURA 3: Comparação entre os modelos de predição do volume utilizados para regular a floresta, em que VPL, V1, V2 e V3 correspondem à produção anual $\left(\mathrm{m}^{3}\right)$, segundo as soluções obtidas por Programação Linear utilizando estimativas de volume dos modelos de Projeção Linear, 1, 2 e 3 respectivamente.

FIGURE 3: Comparison among the models of prediction of the volume used to regulate the forest, in that VPL, V1, V2 and V3 correspond to the annual yield $\left(\mathrm{m}^{3}\right)$, according to the solutions obtained by Linear Programming using estimates of volume of the models of Linear Projection, 1, 2 and 3 , respectively. 
Na Tabela 6, é apresentada uma análise de sensibilidade de cada problema de PL a variações no vetor de custos. Nessa análise, são mostradas variações para cima e para baixo que os coeficientes $c_{i j}$ das variáveis do modelo suportam, de modo que a solução básica não se altere. Como nesse exemplo a solução básica significa o modelo de regulação a ser seguido, essa análise informa indiretamente a sensibilidade de mudanças no modelo de regulação às previsões da produção volumétrica, visto que mudanças no vetor de custos estão diretamente associadas a mudanças nas previsões de volume.

TABELA 6: Análise de sensibilidade para os coeficientes das variáveis de decisão considerando-se os diferentes modelos de produção avaliados.

TABLE 6: Sensibility analysis for the coefficients of the variables of decision being considered the different yield models evaluated.

\begin{tabular}{|c|c|c|c|c|c|c|c|c|c|c|c|c|}
\hline \multirow{2}{*}{ Var. } & \multicolumn{3}{|c|}{ Projeção Linear } & \multicolumn{3}{|c|}{ Modelo 1} & \multicolumn{3}{|c|}{ Modelo 2} & \multicolumn{3}{|c|}{ Modelo 3} \\
\hline & \begin{tabular}{|l|} 
Coef. \\
\end{tabular} & Acres. & Decr. & Coef. & Acres. & Decr. & Coef. & Acres. & Decr. & Coef. & Acres. & Decr. \\
\hline$X_{11}$ & $-124,15$ & 82,55 & Inf. & $-117,95$ & 89,75 & Inf. & $-123,45$ & 85,59 & Inf. & $-147,87$ & 43,44 & Inf. \\
\hline$X_{12}$ & $-48,46$ & 23,00 & 47,29 & $-78,03$ & 53,93 & & $-30,07$ & 48,19 & & $-112,16$ & 5,64 & If. \\
\hline$X_{13}$ & 21,54 & 38,2 & 21,5 & $-54,11$ & 54,11 & Inf. & 51,53 & 3,62 & 18,44 & $-80,50$ & 42,96 & Inf. \\
\hline$X_{21}$ & $-127,75$ & Inf. & 75,04 & $-131,31$ & 19,51 & 48,80 & $-136,70$ & 41,64 & 108,54 & $-155,33$ & 9,97 & 449,02 \\
\hline$K_{22}$ & $-63,23$ & 100,70 & Inf. & $-92,16$ & 59,78 & Inf. & $-45,14$ & 47,17 & 81,19 & $-120,84$ & 43,48 & 12,38 \\
\hline$\tau_{23}$ & $-38,78$ & & Inf. & $-7,23$ & 51,24 & & 26,36 & 108,54 & & $-98,85$ & 24,69 & Inf. \\
\hline$K_{31}$ & -76 , & 15 & 100,9 & 56,16 & & Inf. & 131,13 & 62,04 & Inf. & $-2,45$ & 75,98 & Inf. \\
\hline$X_{32}$ & $-35,2$ & 100,97 & 8,21 & 160,60 & 94,75 & 37,89 & 214,12 & Inf. & 62,04 & 56,65 & Inf. & 21,42 \\
\hline 33 & 22,67 & 166,94 & Inf. & 341,76 & 37,89 & 94,74 & 271,82 & 151,04 & Inf. & 97,72 & 21,42 & Inf. \\
\hline$r_{41}$ & 380 , & Inf. & 68,27 & 359,98 & 230,08 & 78,97 & 488,68 & 101,20 & 12,25 & 375,80 & 21,58 & 33,01 \\
\hline$K_{42}$ & & 68,27 & In & 150 , & 144,98 & Inf. & 204,27 & 84 & Inf. & 109,10 & 17 & Inf. \\
\hline$X_{43}$ & 0,81 & 9,20 & Inf. & 328,92 & 78,97 & 230,08 & 258,83 & 0,71 & 101,20 & 141,16 & 96,27 & 21,58 \\
\hline$X_{51}$ & 207,03 & 10,84 & Inf. & 343,83 & 51,92 & Inf. & 473,84 & 56,74 & Inf. & 327,97 & 32,24 & Inf. \\
\hline$X_{52}$ & 57,44 & 235,57 & Inf. & 428,27 & 288,32 & 51,92 & 542,80 & 32,18 & Inf. & 376,99 & 22,43 & Inf. \\
\hline$X_{53}$ & 2 & 67,40 & 150,17 & 438,89 & ,99 & 288,32 & 543,65 & 14,63 & 32,18 & 373,78 & 37,06 & 22,43 \\
\hline$X_{54}$ & 8,63 & 0,17 & 67,40 & 322,53 & 08,05 & 98,96 & 251,72 & 12 & 14,63 & 117,52 & 362,25 & 37,06 \\
\hline $\mathrm{X}_{61}$ & 7,46 & 77,46 & Inf. & 337,43 & 747,27 & Inf. & 469,64 & 160,21 & Inf. & 455,94 & 727,02 & Inf. \\
\hline$x_{60}$ & 775,15 & 250,31 & Inf. & 416,66 & 670,98 & Inf. & 533,12 & 193,86 & Inf. & 494,02 & 761,05 & Inf. \\
\hline$X_{63}$ & 0,30 & 493,48 & In & 555,84 & 572,55 & Inf. & 581,68 & 194,53 & Inf. & 522,05 & 787,77 & Inf. \\
\hline$X_{64}$ & 7,11 & & Inf. & 427,45 & & Ir &, 25 & 74 & 24,75 &, 02 & 29,73 & 4,18 \\
\hline$X_{6}$ & 18,08 & 39535,43 & 73,89 & 507,71 & 116,53 & Inf. & 546,65 & 24,75 & 1,74 & 526,82 & 4,18 & Inf. \\
\hline$X_{\text {. }}$ & 665,74 & 244,35 & 39535,43 & 600,46 & Inf. & 116,53 & 522,73 & 38,46 & 137,83 & 499,11 & 29,07 & 29,73 \\
\hline$X_{71}$ & 1390,81 & 133,73 & Inf. & 1049,25 & 557,00 & Inf. & 899,82 & 194,23 & Inf. & 1420,27 & 1470,35 & Inf. \\
\hline$X_{72}$ & 07,43 & 52922,66 & 133,73 & 64,68 & 1556,38 & Inf. & 938,79 & 278,76 & Inf. & 1480,70 & 1540,90 & Inf. \\
\hline$X_{73}$ & 95,18 & 210,92 & Inf. & 962,64 & 719,24 & Inf. & 972,49 & 309,25 & Inf. & 1518,95 & 1602,42 & Inf. \\
\hline$X_{7}$ & 1319,72 & 213,87 & 55,61 & 848,37 & Inf. & 35,94 & 907,47 & Inf. & 2,33 & 1421,98 & Inf. & 21,16 \\
\hline$X_{75}$ & 1434,64 & & Inf. & 868,72 & 35,94 & Inf. & 949,83 & 2,33 & Inf. & 1484,40 & 21,16 & Inf. \\
\hline $\mathrm{X}_{81}$ & 1112,05 & 126,08 & 133,59 & 860,44 & 97,11 & 38,83 & 921,17 & Inf. & 86,08 & 1348,83 & 49,52 & 126,96 \\
\hline$X_{82}$ & 4,03 & 33,59 & 6,08 & 875,25 & 38,83 & 97,11 & 958,59 & 86,08 & Inf. & 1476,75 & 8,55 & 60,74 \\
\hline$X_{0}$ & 1068,61 & 214,88 & Inf. & 777,27 & 158,79 & Inf. & 990,94 & 117,92 & Inf. & 1574,69 & 126,96 & 8,29 \\
\hline
\end{tabular}

Em que: Inf. = variação infinita; coef. = coeficiente associado à variável na função objetivo; Acres. = acréscimos permitidos para que a base permaneça a mesma; Decr. = decréscimos permitidos para que a base permaneça a mesma.

Pode-se observar, na Tabela 6, que as variáveis da base, de maneira geral, são muito sensíveis a mudanças em seus coeficientes, ou seja, caso o valor econômico associado a essas variáveis sofra pequenas variações, essas variáveis podem deixar a base, alterando a forma de se regular a floresta. Com isso, pode-se concluir que pequenas variações na predição da produção em volume, as quais são utilizadas para gerar os resultados econômicos, podem levar a diferentes maneiras de se conduzir a floresta. 
O que influenciou diretamente as diferentes respostas para as soluções dos problemas de PL resolvidos com diferentes estimativas de produção foram, sem dúvida, as diferentes combinações dos vetores de custos e das matrizes de coeficientes tecnológicos. Com vistas a estudar apenas o impacto da matriz de coeficientes tecnológicos, os problemas de PL anteriores foram resolvidos novamente, porém, utilizando-se uma mesma função objetivo para todos os problemas PL, variando-se, portanto, a matriz dos coeficientes tecnológicos. Embora se saiba que mudanças nessa matriz provocam mudanças na função objetivo, decidiu-se por fixar uma única função objetivo para diferentes matrizes de coeficientes tecnológicos com o único intuito de demonstrar que variações ocorridas nos coeficientes tecnológicos, nesse caso os coeficientes de produção volumétrica, podem alterar todo o processo de tomada de decisão. A forma encontrada para isolar o efeito dos coeficientes volumétricos de produção foi variá-los utilizando as estimativas de produção obtidas pelos modelos ajustados e manter constantes os demais coeficientes tecnológicos e a função objetivo. Nesse caso, decidiu-se utilizar a função objetivo gerada pelo modelo 3, por ser considerada, além de precisa, exequível para qualquer tipo de floresta eqüiânea. Os resultados são apresentados na Tabela 7.

TABELA 7: Comparação das respostas do modelo de Programação Linear considerando uma mesma função objetivo e diferentes matrizes de coeficientes tecnológicos, conforme os modelos de produção avaliados.

TABLE 7: Comparison of the answers of the Linear Programming models considering the same objective function and different matrices of technological coefficients, according to yield models evaluated.

\begin{tabular}{|c|c|c|c|c|}
\hline \multirow{2}{*}{ Var. } & Projeção Linear & Modelo 1 & Modelo 2 & Modelo 3 \\
\hline & $\mathrm{FO}=\mathrm{R} \$ 169.555,20$ & $\mathrm{FO}=\mathrm{R} \$ 181.821,70$ & $\mathrm{FO}=\mathrm{R} \$ 179.771,50$ & $\mathrm{FO}=\mathrm{R} \$ 160.207,50$ \\
\hline $\mathrm{X}_{11}$ & 0,00 & 0,00 & 0,00 & 0,00 \\
\hline $\mathrm{X}_{12}$ & 4,63 & 0,00 & 0,00 & 0,00 \\
\hline $\mathrm{X}_{13}$ & 0,00 & 0,00 & 0,00 & 0,00 \\
\hline $\mathrm{X}_{21}$ & 82,02 & 44,10 & 13,26 & 53,85 \\
\hline$X_{22}$ & 2,98 & 0,00 & 71,74 & 27,84 \\
\hline$X_{23}$ & 0,00 & 0,00 & 0,00 & 0,00 \\
\hline$X_{31}$ & 0,00 & 0,00 & 0,00 & 0,00 \\
\hline$X_{32}$ & 50,00 & 38,26 & 50,00 & 50,00 \\
\hline$X_{33}$ & 0,00 & 11,74 & 0,00 & 0,00 \\
\hline $\mathrm{X}_{41}$ & 50,00 & 37,63 & 36,36 & 40,65 \\
\hline$X_{42}$ & 0,00 & 0,00 & 0,00 & 0,00 \\
\hline $\mathrm{X}_{43}$ & 0,00 & 12,37 & 13,64 & 9,35 \\
\hline $\mathrm{X}_{51}$ & 0,00 & 0,00 & 0,00 & 0,00 \\
\hline$X_{52}$ & 0,00 & 0,00 & 0,00 & 0,00 \\
\hline $\mathrm{X}_{53}$ & 4,11 & 32,39 & 27,03 & 13,55 \\
\hline$X_{54}$ & 85,89 & 57,61 & 62,97 & 76,45 \\
\hline$X_{61}$ & 0,00 & 0,00 & 0,00 & 0,00 \\
\hline $\mathrm{X}_{62}$ & 0,00 & 0,00 & 0,00 & 0,00 \\
\hline$X_{63}$ & 0,00 & 0,00 & 0,00 & 0,00 \\
\hline$X_{64}$ & 3,45 & 23,69 & 22,44 & 6,07 \\
\hline$X_{65}$ & 5,96 & 0,00 & 0,00 & 0,00 \\
\hline $\mathrm{X}_{66}$ & 25,59 & 11,31 & 12,56 & 28,93 \\
\hline$X_{71}$ & 2,19 & 0,00 & 0,00 & 0,00 \\
\hline$X_{72}$ & 0,00 & 0,00 & 0,00 & 0,00 \\
\hline$X_{73}$ & 0,00 & 0,00 & 0,00 & 0,00 \\
\hline $\mathrm{X}_{74}$ & 32,81 & 0,00 & 35,00 & 35,00 \\
\hline$X_{75}$ & 0,00 & 35,00 & 0,00 & 0,00 \\
\hline $\mathrm{X}_{81}$ & 47,17 & 44,76 & 24,88 & 39,02 \\
\hline $\mathrm{X}_{82}$ & 12,83 & 6,97 & 12,33 & 8,32 \\
\hline$X_{83}$ & 0,00 & 8,27 & 22,79 & 4,05 \\
\hline
\end{tabular}

Em que: $\mathrm{FO}=$ valor obtido para a função objetivo para cada modelo. 
De acordo com essa tabela, pode-se verificar que, para uma mesma função objetivo, diferentes respostas para os problemas de PL foram encontradas, comprovando-se a importância da matriz de coeficientes tecnológicos na tomada de decisão, representada, nesse caso, sobretudo pelas informações da produção. Verifica-se, portanto, que não apenas na determinação dos resultados econômicos, mas também no atendimento das restrições, estimativas precisas de volume são importantes.

Analisando as Figuras 4 e 5, que representam as respostas encontradas na Tabela 7, podem-se verificar as diferenças entre as estratégias de regulação, especialmente em termos de área de corte, quando se variaram os dados da matriz tecnológica fixando-se a função objetivo.

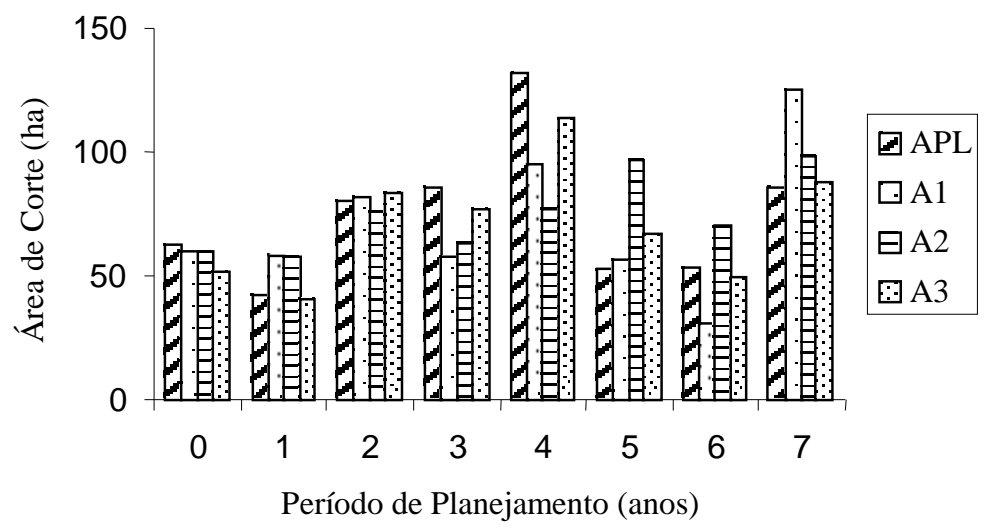

FIGURA 4: Comparação entre os modelos de predição do volume utilizados para regular a floresta, em que APL, A1, A2 e A3 correspondem às áreas anuais cortadas (ha), segundo as soluções obtidas por Programação Linear empregando estimativas de volume dos modelos de Projeção Linear, 1, 2 e 3, respectivamente, para uma mesma função objetivo.

FIGURE 4: Comparison among the models of prediction of the volume used to regulate the forest, in that APL, A1, A2 and A3 correspond to the annual area cut (ha), according to the solutions obtained by Linear Programming using estimates of volume of the models of Linear Projection, 1, 2 and 3, respectively, for the same objective function.

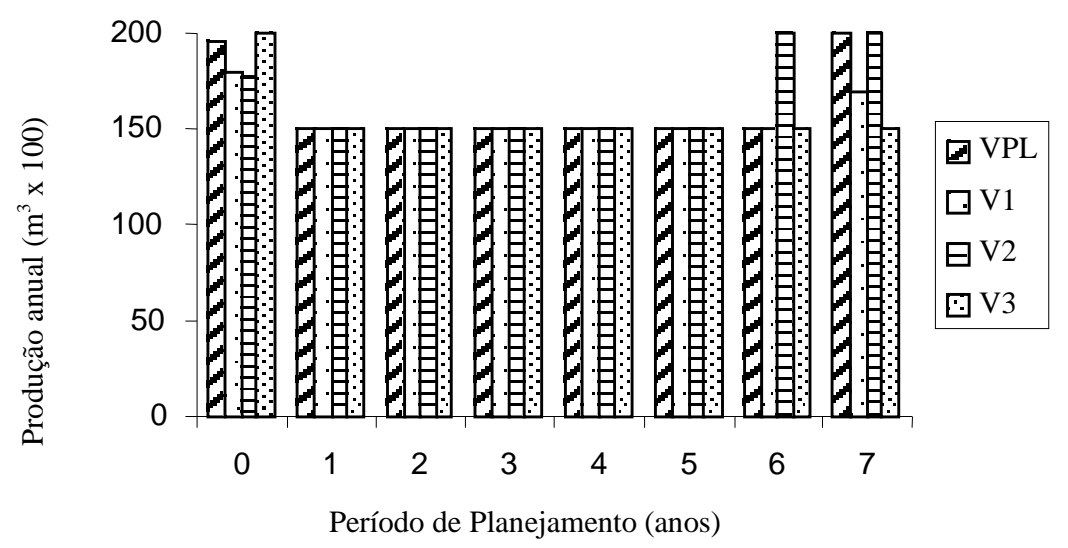

FIGURA 5: Comparação entre os modelos de predição do volume utilizados para regular a floresta, em que VPL, V1, V2 e V3 correspondem à produção anual $\left(\mathrm{m}^{3}\right)$, segundo as soluções obtidas por Programação Linear utilizando estimativas de volume dos modelos de Projeção Linear, 1, 2 e 3 respectivamente para uma mesma função objetivo.

FIGURE 5: Comparison among the models of prediction of the volume used to regulate the forest, in that VPL, V1, V2 and V3 correspond to the annual yield $\left(\mathrm{m}^{3}\right)$, according to the solutions obtained by Linear Programming using estimates of volume of the models of Linear Projection, 1, 2 and 3 , respectively, for the same objective function.

Ciência Florestal, v. 13, n. 1, 2003 
Weintraub e Abramovich (1995), ao estudarem os efeitos de incertezas das produções futuras no manejo florestal, concluíram que não considerar incertezas em modelos cujas restrições de demanda têm pouca folga, pode conduzir a situações de manejo com soluções inviáveis, reforçando a idéia de que quanto mais incertos ou imprecisos forem os dados dos coeficientes tecnológicos em termos de volume, mais significativas podem ser as conseqüências no processo de tomada de decisão.

Numerosos modelos estão disponíveis para predizer o crescimento e a produção florestal. Avaliar qualquer um desses modelos depende dos objetivos e das restrições do manejador. Com base nessas preocupações, Buchman e Shifley (1983) discutiram em seu trabalho aspectos práticos para a escolha de sistemas de predição para fins de manejo, propondo um guia básico para escolha de sistemas de predição. Esse guia considerou características como exatidão, precisão, dependência do tempo, custo, realismo biológico, flexibilidade e simplicidade.

Muitos estudos ainda carecem de ser feitos, procurando associar as questões da modelagem de sistemas de predição às questões de planejamento. Assim, conforme demonstrado neste trabalho, não se pode imaginar a dissociação de sistemas de predição, avaliações econômicas e sistemas de planejamento, fazendo todos parte de um mesmo contexto. O tratamento isolado de um desses elementos pode levar a um fracasso do modelo geral, por se ignorar que esses elementos têm forte relacionamento entre si. Metodologias de sistemas de informação parecem um promissor campo de pesquisa para contextualizar os elementos citados, de modo a valorizar o fluxo da informação e otimizar de maneira global o desempenho do processo de tomada de decisão. Finalmente, considerando o papel estratégico fundamental exercido pelas informações sobre a produção florestal, maior atenção deveria ser dada ao uso de sistemas de predição no Brasil, tanto no sentido da realização de novas pesquisas como no investimento no uso de modelos que realmente sejam adequados aos objetivos de manejo traçados.

Ainda assim, a avaliação de sistemas de predição é algo complexo e até certo ponto subjetivo. Com o intuito de tornar mais objetiva essa avaliação, Brand e Holdaway (1983) propuseram testes de avaliação e validação de sistemas de projeção, tratando em especial questões de exatidão, precisão e dependência do tempo. Esses autores consideraram que o sucesso de sistemas de projeção está intimamente ligado aos objetivos ao qual o sistema se destina. Dias (2000) avaliou modelos de crescimento e produção em nível de povoamento total em duas etapas, sendo, na primeira, empregada a análise estatística dos resíduos, conforme procedimentos aferidos por Guimarães (1994), para avaliação dos métodos de construção de índices de local, juntamente com a análise de distribuição dos resíduos. A segunda etapa referiu-se aos fundamentos biológicos do modelo por meio da análise de consistência das estimativas em relação aos efeitos de área basal inicial e do índice de local sobre a idade de corte.

Nos trabalhos de Soares et al. (1998a, 1998b, 1998c), é apresentado um sistema de predição para eucalipto em que foram propostos um intervalo de previsão para obtenção de estimativas mínimas confiáveis e o estudo da periodicidade e da probabilidade de ocorrência de variações dos elementos climáticos, de modo a dar subsídios às tomadas de decisão em projetos implantados, bem como fornecer estimativas de risco para investimentos futuros. Dadas as incertezas da produção e suas influências em modelos de planejamento, essa linha de pesquisa se mostra promissora no sentido de aumentar a confiança das estimativas de volume e permitir a simulação de cenários de produção e seus possíveis impactos no modelo de planejamento. Modelos ecológicos também aparecem como tendência promissora e podem incorporar mais confiabilidade ao processo de predição da produção, embora ainda tenham uso restrito, em razão do seu elevado custo de implementação.

\section{CONCLUSÕES}

As principais conclusões deste trabalho foram:

Modelos de regulação florestal cujas estimativas de produção foram obtidas por diferentes modelos de prognose da produção, quando resolvidos por Programação Linear, podem levar a diferentes maneiras de se conduzir a floresta, valorizando-se, portanto, a escolha de modelos de prognose da produção mais precisos.

O vetor de custos do modelo de Programação Linear proposto para regular a floresta mostrou-se bastante sensível a variações em seus coeficientes, sugerindo que as predições da produção em volume influenciam de forma significativa o processo de tomada de decisão. 
Ao se isolar o efeito da matriz de coeficientes tecnológicos, alimentado-a com diferentes estimativas de produção e para uma mesma função objetivo, mostrou-se que ela pode afetar de maneira significativa o processo de regulação da floresta.

\section{REFERÊNCIAS BIBLIOGRÁFICAS}

BRAND, G.J.; HOLDAWAY, M.R. Users need performance information to evaluate models. Journal of Forestry, v. 81, p. 235-237, 1983.

BREGALDA, P.F.; OLIVEIRA, A.A.F; BORNSTEIN, C.T. Introdução à programação linear. 3. ed. Rio de Janeiro: Campus, 1988. 329p.

BUCHMAN, R.G.; SHIFLEY, S.R. Guide to evaluating forest growth projection systems. Journal of Forestry, v. 81, p. 232-234, 1983.

CAMPOS, J.C.C.; ROSAS, M.P.; LEITE, H.G. Comparação de alternativas de determinação da idade técnica de corte em plantações de eucalipto. Revista Árvore, Viçosa-MG, v. 20, n. 1, p. 37-49, 1996.

CLUTTER, J.L. Compatible growth and yields models for loblolly pine. Forest Science, v. 9, n. 3, p. 354-371, 1963.

CLUTTER, J.L.; PIENAAR, L.V.; BRISTER, G.H. et al. Timber management: a quantitative approach. 3. ed. New York: Jonh Willey, 1983. 333p.

COUTO, H.T.Z.; BASTOS, N.L.M. Curvas de crescimento em volume para Eucalyptus em $2^{\mathrm{a}}$ rotação no estado de São Paulo. IPEF, Piracicaba, v. 34, p. 15-21, 1986.

DAVIS, L.S., JOHNSON, K.N. Forest management. 3.ed. New York: Mc Graw- Hill Book Company, 1987. 790p.

DIAS, A.N. Modelagem e avaliação econômica de plantações de eucalipto submetidas a desbastes. 2000. 70p. Dissertação (Mestrado em Ciência Florestal) - Universidade Federal de Viçosa, Viçosa, 2000.

DYKSTRA, D.P. Mathematical programming for natural resource management. New York: McGraw-Hill, 1984. 318p.

GOMES, F.S.; MAESTRI, R.; SANQUETA, C.R. Análise financeira de regimes de manejo em povoamentos de Pinus taeda L., visando a produção de madeira para a indústria de papel e celulose. Revista Árvore, Viçosa, v. 22, n. 2, p. 227-243, 1998.

GUIMARÃES, D.P. Desenvolvimento de um modelo de distribuição diamétrica de passo invariante para prognose e projeção da estrutura de povoamentos de eucalipto. 1994. 160p. Tese (Doutorado em Ciência Florestal) Universidade Federal de Viçosa, Viçosa, 1994.

JOHNSON, K.N.; SCHEURMAN, H.L. Techiniques for prescribing optimal timber harvest and investment under different objectives - discussion and synthesis. Forest Science, Washington, v. 18, n. 1, p. 1-31, 1977.

REZENDE, J.L.P.; OLIVEIRA, A.D. Avaliação de projetos florestais. Viçosa: Imprensa Universitária, UFV, 1993. 49p.

RIBEIRO, C.A.A.S. Pesquisa operacional aplicada ao manejo florestal. Viçosa, MG: UFV, Departamento de Engenharia Florestal, 1996. 107p. (Notas de aula).

SOARES, C.P.B.; LEITE, H.G.; CAMPOS, J.C.C.; SEDIYAMA, G.C. Intervalo de previsão para um modelo de crescimento e produção composto por equações simultâneas. Revista Árvore, Viçosa-MG, v. 22, n. 2, p. 185-192, 1998a.

SOARES, C.P.B.; LEITE, H.G.; SEDIYAMA, G.C. Caracterização da periodicidade e da probabilidade de ocorrência de precipitações anuais como subsídios para o planejamento florestal. Revista Árvore, Viçosa-MG, v. 22, n. 3, p. 315-324, 1998b.

SOARES, C.P.B.; LEITE, H.G.; CAMPOS, J.C.C.; SEDIYAMA, G.C. A inclusão da precipitação anual em um modelo de crescimento e produção. Revista Árvore, Viçosa-MG, v. 22, n. 4, p. 475-482, 1998 c.

SULIVAN, A.D.; CLUTTER, J.L. Simultaneous growth and yield model for loblolly pine. Forest Science, v. 18, n. 1, p. 76-86, 1972.

TREVISOL JÚNIOR, T.L. Análise de um modelo compatível de crescimento e produção de Eucalyptus grandis (W. Hill ex Maiden). 1985. 74p. Dissertação (Mestrado em Ciência Florestal) - Universidade Federal de Viçosa, Viçosa, 1985 .

VOLPI, N.M.P.; CARNIERI, C.; SANQUETA, C.R. O impacto da estocasticidade das informações em um modelo de planejamento florestal. Revista Árvore, Viçosa-MG, v. 12, n. 2, p. 100-110, 1999.

WEINTRAUB, A.; ABRAMOVICH, A. Analysis of uncertainty of future timber yields in forest management. Forest Science, v. 41, n. 2, p. 217-234, 1995.

Ciência Florestal, v. 13, n. 1, 2003 